\title{
Palladium-catalyzed phosphine-, copper-free and aerobic Sonogashira coupling in aqueous media
}

\author{
Chun Liu, * Fanying Bao, and Qijian Ni \\ State Key Lab of Fine Chemicals, Dalian University of Technology, Dalian 116024, P R China \\ E-mail: chunliu70@yahoo.com
}

\begin{abstract}
A simple, highly efficient and phosphine-free protocol for the Sonogashira coupling of aryl iodides with terminal alkynes has been developed using $\operatorname{Pd}_{2}(\mathrm{dba})_{3}(\mathrm{dba}=$ dibenzylideneacetone) as the catalyst under aqueous, copper-free and aerobic conditions. The coupling of aryl iodides with aromatic terminal alkynes provided good to excellent yields and moderate to good yields were achieved using aliphatic terminal alkynes as one of the coupling partners. Aqueous ethanol as solvent is economical and environmentally benign in accordance with the concept of green chemistry.
\end{abstract}

Keywords: Palladium, phosphine-free, aerobic, Sonogashira coupling, aqueous

\section{Introduction}

The Sonogashira cross-coupling reaction has become one of the most important methods in the formation of carbon-carbon bonds over the past three decades, which plays an important role in the synthesis of pharmaceuticals, agrochemicals and functional materials. ${ }^{1}$ Generally, this reaction proceeds in the presence of phosphine ligands with the combination of palladium and copper salts using a large excess of amine as base or solvent. ${ }^{1 \mathrm{a}, 2}$ However, the copper salts as the co-catalysts would induce homocoupling reaction of terminal alkynes. ${ }^{3}$ Moreover, phosphine ligands are also sensitive to air or moisture, which restricts this reaction to proceed only under an inert atmosphere. To solve these problems, efforts have been made including the employment of sole palladium salts as the catalysts (copper-free systems), ${ }^{4}$ reducing atmosphere $\left(\mathrm{H}_{2}\right)^{5}$ (phosphine ligands were still used) and palladium complexes ${ }^{6}$ containing air-stable ligands such as $N$-heterocyclic carbene ligands. ${ }^{7}$ Although these palladium complexes of air-stable ligandsinvolved protocols delivered the corresponding products successfully, most of the ligands or palladium complexes prepared via multi-synthesis were expensive. Noticeably, among recent advances on this topic, phosphine-free protocols ${ }^{8}$ have obtained attention because of its 
simplicity and economy in comparison with the ligand-promoted ones, such as $\mathrm{Pd}(\mathrm{OAc})_{2}$ in $\mathrm{DMF}^{8 \mathrm{a}} \mathrm{PdCl}_{2}$ in $\mathrm{H}_{2} \mathrm{O},{ }^{8 \mathrm{~b}} \mathrm{Pd}(\mathrm{OAc})_{2}$ in aqueous acetone, ${ }^{8 \mathrm{~d}} \mathrm{Pd} / \mathrm{C}$ in aqueous isopropanol. ${ }^{8 \mathrm{f}}$

With the enhancement of people's awareness of environment, water-involved Sonogashira reaction has mushroomed. ${ }^{6 f, g, 8 b-d, 8 f, 9}$ In comparison with organic solvent, water is an ideal alternative for organic synthesis due to its non-toxic, incombustibility as well as inexpensiveness, which is of great interest to chemists. Very recently, we reported a palladium-catalyzed phosphine-free protocol for the Suzuki reaction in aqueous ethanol. ${ }^{10}$ To the best of our knowledge, the palladium-catalyzed phosphine-free Sonogashira reaction in aqueous ethanol has not been reported. In the present paper, we intend to develop an aerobic and water-involved phosphine-free system for the palladium-catalyzed Sonogashira reaction under mild and copperfree conditions.

\section{Results and Discussion}

\section{Investigation of reaction conditions}

Initially the coupling of 4-iodoanisole and phenylacetylene was chosen as the model reaction for screening the reaction conditions. The corresponding results are collected in Table 1. Firstly, the effects of solvents on this Sonogashira reaction were investigated. Obviously, the coupling in water with ethanol as the co-solvent provided the highest isolated yield than those with other organic solvents under the same amount of water (Table 1, entry 4). The coupling in aqueous glycol was hardly to obtain the desired product (Table 1, entry 2). Only a $26 \%$ yield was obtained with respect to DMF as the co-solvent (Table 1, entry 1).

It was noteworthy that the amount of water had a marked effect on the coupling of the 4iodoanisole with phenylacetylene (Table 1, entries 4-8). Only a 44\% yield was obtained when the reaction proceeded in pure ethanol (Table 1 , entry 5). Increasing the water content to $25 \%$, the coupling gave $91 \%$ yield (Table 1, entry 6). With further increasing the water content to $60 \%$, the yield was decreased to $28 \%$ (Table 1 , entry 7 ). When the water content accounted for three fourths of solvent, the yield was sharply decreased to trace (Table 1, entry 8). Therefore, the suitable volume ratio of ethanol to water is $3: 1$.

Next, we investigated the effects of precatalysts on this reaction. Precatalysts like $\mathrm{Pd}(\mathrm{OAc})_{2}$, $\mathrm{Pd} / \mathrm{C}, \mathrm{Pd}_{2}(\mathrm{dba})_{3}$ had the similar catalytic effect in comparison with the $\mathrm{PdCl}_{2}$ which afforded $81 \%$ isolated yield (Table 1, entries 6, 9, 10 and 11$). \operatorname{Pd}(\mathrm{OAc})_{2}$ and $\mathrm{Pd}_{2}(\mathrm{dba})_{3}$ are non-supported catalysts, while $\mathrm{Pd} / \mathrm{C}$ is a supported catalyst. Gratifying, they all could provide good results. More interesting, $\mathrm{Pd} / \mathrm{C}$ has the advantages such as comercial availability, low cost, easy recycle by filtration. $\mathrm{Pd}_{2}(\mathrm{dba})_{3}$ provided the highest yield and was chosen as the optimal precatalyst for further investigaion.

Through screening different bases, it was found that $\mathrm{K}_{3} \mathrm{PO}_{4} \cdot 7 \mathrm{H}_{2} \mathrm{O}$ and $\mathrm{NaOH}$ could afford good to excellent yields (Table 1, entries 11 and 12), while both $\mathrm{K}_{2} \mathrm{CO}_{3}$ and organic base $\mathrm{Et}_{3} \mathrm{~N}$ 
delivered moderate yields (Table 1, entries 13 and 14). The results demonstrated that $\mathrm{K}_{3} \mathrm{PO}_{4} \cdot 7 \mathrm{H}_{2} \mathrm{O}$ was the suitable base to activate this catalytic system.

The temperature was also an important factor for the reaction. Lowering the temperature significantly reduced the yields of the coupling products. For example, when lowering the temperature to $50{ }^{\circ} \mathrm{C}$ and $25{ }^{\circ} \mathrm{C}$, the coupling products were delivered in $72 \%$ and $10 \%$ yields, respectively (Table 1 , entries 15 and 16). Therefore, $80{ }^{\circ} \mathrm{C}$ was chosen as the optimal temperature.

Table 1. Screening of the optimum conditions for the Sonogashira coupling of 4-iodoanisole with phenylacetylene ${ }^{\mathrm{a}}$

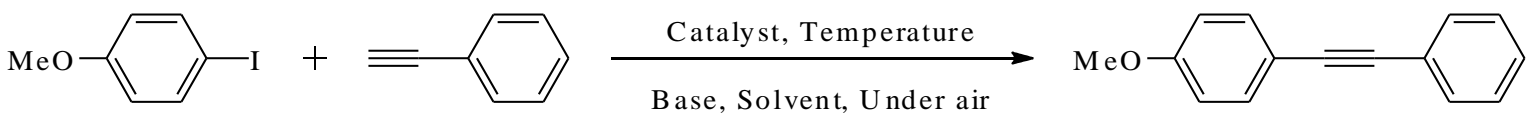

\begin{tabular}{|c|c|c|c|c|c|}
\hline Entry & Catalyst & Base & Solvent (4mL) & Time (min) & Yield $(\%)^{\mathrm{b}}$ \\
\hline 1 & $\mathrm{Pd}(\mathrm{OAc})_{2}$ & $\mathrm{~K}_{3} \mathrm{PO}_{4} \cdot 7 \mathrm{H}_{2} \mathrm{O}$ & $\mathrm{DMF} / \mathrm{H}_{2} \mathrm{O}(2 / 2)$ & 60 & 26 \\
\hline 2 & $\mathrm{Pd}(\mathrm{OAc})_{2}$ & $\mathrm{~K}_{3} \mathrm{PO}_{4} \cdot 7 \mathrm{H}_{2} \mathrm{O}$ & Glycol/ $\mathrm{H}_{2} \mathrm{O}(2 / 2)$ & 60 & trace \\
\hline 3 & $\mathrm{Pd}(\mathrm{OAc})_{2}$ & $\mathrm{~K}_{3} \mathrm{PO}_{4} \cdot 7 \mathrm{H}_{2} \mathrm{O}$ & $i-\mathrm{PrOH} / \mathrm{H}_{2} \mathrm{O}(2 / 2)$ & 60 & 73 \\
\hline 4 & $\mathrm{Pd}(\mathrm{OAc})_{2}$ & $\mathrm{~K}_{3} \mathrm{PO}_{4} \cdot 7 \mathrm{H}_{2} \mathrm{O}$ & $\mathrm{EtOH} / \mathrm{H}_{2} \mathrm{O}(2 / 2)$ & 60 & 77 \\
\hline 5 & $\mathrm{Pd}(\mathrm{OAc})_{2}$ & $\mathrm{~K}_{3} \mathrm{PO}_{4} \cdot 7 \mathrm{H}_{2} \mathrm{O}$ & $\mathrm{EtOH}$ & 60 & 44 \\
\hline 6 & $\mathrm{Pd}(\mathrm{OAc})_{2}$ & $\mathrm{~K}_{3} \mathrm{PO}_{4} \cdot 7 \mathrm{H}_{2} \mathrm{O}$ & $\mathrm{EtOH} / \mathrm{H}_{2} \mathrm{O}(3 / 1)$ & 60 & 91 \\
\hline 7 & $\mathrm{Pd}(\mathrm{OAc})_{2}$ & $\mathrm{~K}_{3} \mathrm{PO}_{4} \cdot 7 \mathrm{H}_{2} \mathrm{O}$ & $\mathrm{EtOH} / \mathrm{H}_{2} \mathrm{O}(2 / 3)$ & 60 & 28 \\
\hline 8 & $\mathrm{Pd}(\mathrm{OAc})_{2}$ & $\mathrm{~K}_{3} \mathrm{PO}_{4} \cdot 7 \mathrm{H}_{2} \mathrm{O}$ & $\mathrm{EtOH} / \mathrm{H}_{2} \mathrm{O}(1 / 3)$ & 60 & trace \\
\hline 9 & $\mathrm{PdCl}_{2}$ & $\mathrm{~K}_{3} \mathrm{PO}_{4} \cdot 7 \mathrm{H}_{2} \mathrm{O}$ & $\mathrm{EtOH} / \mathrm{H}_{2} \mathrm{O}(3 / 1)$ & 60 & 81 \\
\hline 10 & $\mathrm{Pd} / \mathrm{C}(5 \%)$ & $\mathrm{K}_{3} \mathrm{PO}_{4} \cdot 7 \mathrm{H}_{2} \mathrm{O}$ & $\mathrm{EtOH} / \mathrm{H}_{2} \mathrm{O}(3 / 1)$ & 60 & 90 \\
\hline 11 & $\mathrm{Pd}_{2}(\mathrm{dba})_{3}$ & $\mathrm{~K}_{3} \mathrm{PO}_{4} \cdot 7 \mathrm{H}_{2} \mathrm{O}$ & $\mathrm{EtOH} / \mathrm{H}_{2} \mathrm{O}(3 / 1)$ & 60 & 93 \\
\hline 12 & $\mathrm{Pd}_{2}(\mathrm{dba})_{3}$ & $\mathrm{NaOH}$ & $\mathrm{EtOH} / \mathrm{H}_{2} \mathrm{O}(3 / 1)$ & 40 & 82 \\
\hline 13 & $\mathrm{Pd}_{2}(\mathrm{dba})_{3}$ & $\mathrm{Et}_{3} \mathrm{~N}$ & $\mathrm{EtOH} / \mathrm{H}_{2} \mathrm{O}(3 / 1)$ & 60 & 42 \\
\hline 14 & $\mathrm{Pd}_{2}(\mathrm{dba})_{3}$ & $\mathrm{~K}_{2} \mathrm{CO}_{3}$ & $\mathrm{EtOH} / \mathrm{H}_{2} \mathrm{O}(3 / 1)$ & 60 & 34 \\
\hline $15^{\mathrm{c}}$ & $\mathrm{Pd}_{2}(\mathrm{dba})_{3}$ & $\mathrm{~K}_{3} \mathrm{PO}_{4} \cdot 7 \mathrm{H}_{2} \mathrm{O}$ & $\mathrm{EtOH} / \mathrm{H}_{2} \mathrm{O}(3 / 1)$ & 60 & 72 \\
\hline $16^{\mathrm{d}}$ & $\mathrm{Pd}_{2}(\mathrm{dba})_{3}$ & $\mathrm{~K}_{3} \mathrm{PO}_{4} \cdot 7 \mathrm{H}_{2} \mathrm{O}$ & $\mathrm{EtOH} / \mathrm{H}_{2} \mathrm{O}(3 / 1)$ & 60 & 10 \\
\hline
\end{tabular}

${ }^{a}$ Reaction conditions: 4-iodoanisole $(0.5 \mathrm{mmol})$, phenylacetylene $(0.75 \mathrm{mmol})$, base $(1 \mathrm{mmol})$, catalyst amount $(0.5 \mathrm{~mol} \%), 80{ }^{\circ} \mathrm{C}$, under air. The reactions were monitored by TLC. ${ }^{\mathrm{b}}$ Isolated yields. ${ }^{\mathrm{c}} 50{ }^{\circ} \mathrm{C} .{ }^{\mathrm{d}} 25^{\circ} \mathrm{C}$.

\section{Scope and limitation of substrates}

In order to investigate the feasibility of this aqueous catalytic system, a variety of aromatic terminal alkynes were subjected to the optimum conditions $\left\{0.5 \mathrm{~mol} \% \operatorname{Pd}_{2}(\mathrm{dba})_{3}, \mathrm{EtOH} / \mathrm{H}_{2} \mathrm{O}\right.$ (3/1 in volume), $1 \mathrm{mmol} \mathrm{K}_{3} \mathrm{PO}_{4} \cdot 7 \mathrm{H}_{2} \mathrm{O}, 80{ }^{\circ} \mathrm{C}$, under air\} via the Sonogashira reaction with aryl 
iodides. The results are summarized in Table 2. Good to excellent yields were afforded for the couplings of aryl iodides with a series of substituted aromatic terminal alkynes under this phosphine-free aqueous system. It was clear that aryl iodides bearing electron-withdrawing groups completed the couplings in shorter time than those bearing electron-donating groups. The couplings of electron-rich 4-iodoanisole with phenylacetylene and 4-tolylacetylene provided the products in 93\% and 90\% yields in $1 \mathrm{~h}$, respectively (Table 2, entries 1 and 2), which were more efficient than those in the reported phosphine-free protocols. ${ }^{4 e, 8 a-d, 8 f}$ It was worth noting that using 1-ethynyl-4-methoxybenzene as the coupling partner, 1,2-bis(4-methoxyphenyl)ethyne was obtained in $91 \%$ yield within $45 \mathrm{~min}$, resulting in a TOF value of $243 \mathrm{~h}^{-1}$ (Table 2, entry 3). Nevertheless, 4-fluorophenylacetylene conducted the reaction in $81 \%$ yield in $2 \mathrm{~h}$ (Table 2, entry 4). The coupling of 4-iodoanisole with 3-ethynylaniline provided a 95\% yield after 90 min (Table 2, entry 5). Notably, in the case of electron-poor 4'-iodoacetophenone, the couplings with various substituted aromatic terminal alkynes delivered the corresponding products rapidly in high yields (Table 2, entries 6-10), which were also far more efficient than those in reported phosphine-free systems. ${ }^{8 \mathrm{a}-\mathrm{d},}{ }^{8 \mathrm{f}}$ For example, the coupling of 4 '-iodoacetophenone with phenylacetylene delivered a $96 \%$ yield in 20 min (Table 2, entry 6). Notably, 95\% yield was achieved within 15 min using 1-ethynyl-4-methoxybenzene as the coupling partner (Table 2, entry 8). The Sonogashira reaction between electron-poor 4'-iodoacetophenone and 3ethynylaniline delivered the cross-coupling products in $93 \%$ yield after only 10 min, resulting in a TOF of $1116 \mathrm{~h}^{-1}$ (Table 2, entry 10). The steric effect on the ortho-substituted 2-iodoanisole decreased the reactivity of the couplings with a series of aromatic terminal alkynes, consequently, relative lower yields were obtained with phenylacetylene, 4-tolylacetylene and 3ethynylaniline (Table 2, entries 11-13).

To further investigate the scope and limitation of this phosphine-free aqueous system, a series of aliphatic terminal alkynes were coupled with 4-iodoanisole or 4'-iodoacetophenone. The results are collected in Table 3. Compared with the aromatic terminal alkynes, the coupling of aliphatic alkynes with the electron-rich 4-iodoanisole gave moderate yields (Table 3, entries 1, 3, $5,7)$. The improved yields were obtained when $\mathrm{NaOH}$ was used in place of $\mathrm{K}_{3} \mathrm{PO}_{4} \cdot 7 \mathrm{H}_{2} \mathrm{O}($ Table 3 , entries $2,4,6,8)$. According to the reported literature, ${ }^{1 \mathrm{~g}}$ the reactivity order seems to be partially related to the acidity of the alkynes, generally, the acidity of aromatic alkynes is stronger than the aliphatic alkynes. So $\mathrm{NaOH}$ as a strong base may be favorable for the coupling of aliphatic alkynes. Notably, the electron-poor 4'-iodoacetophenone as the coupling partner provided the products in excellent yields in short time (Table 3, entries 9-11), therefore showing high efficiency. For example, hex-5-ynenitrile and 5-chloropent-1-yne as the coupling partners gave the corresponding cross-coupling products in $92 \%$ and $95 \%$ yields within 20 min, respectively (Table 3, entries 9, 10). Noticeably, when but-3-yn-1-ol as the cross-coupling partner, the 1-(4-(4-hydroxybut-1-yn-1-yl)phenyl)ethanone was achieved in $90 \%$ yield in 15 min, which was more efficient than the aqueous $i$-propanol system (Table 3 , entry 11). ${ }^{8 \mathrm{c}, 8 \mathrm{f}}$ 
Table 2. Sonogashira reaction of aryl iodides with aromatic terminal alkynes ${ }^{\mathrm{a}}$

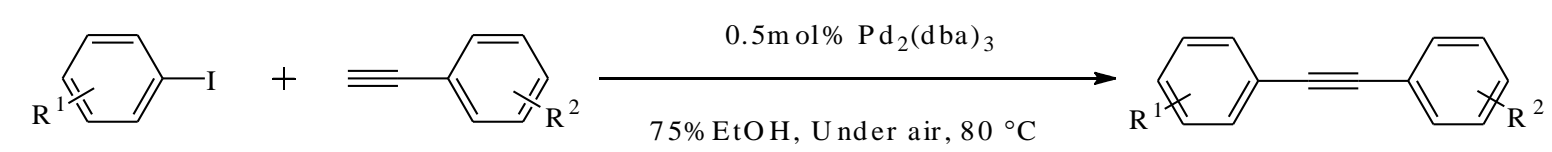

\begin{tabular}{|c|c|c|c|c|c|}
\hline Entry & $\mathrm{R}^{1}$ & $\mathrm{R}^{2}$ & Product & Time(min) & Yield $(\%)^{\mathrm{b}}$ \\
\hline 1 & 4-OMe & $\mathrm{H}$ & & 60 & 93 \\
\hline 2 & 4-OMe & $4-\mathrm{Me}$ & & 60 & 90 \\
\hline 3 & 4-OMe & 4-OMe & & 45 & 91 \\
\hline 4 & 4-OMe & $4-\mathrm{F}$ & & 120 & 81 \\
\hline 5 & 4-OMe & $3-\mathrm{NH}_{2}$ & & 90 & 95 \\
\hline 6 & 4-COMe & $\mathrm{H}$ & & 20 & 96 \\
\hline 7 & 4-COMe & 4-Me & & 18 & 96 \\
\hline 8 & 4-COMe & 4-OMe & & 15 & 95 \\
\hline 9 & 4-COMe & $4-\mathrm{F}$ & & 30 & 91 \\
\hline 10 & 4-COMe & $3-\mathrm{NH}_{2}$ & & 10 & 93 \\
\hline 11 & 2-OMe & $\mathrm{H}$ & & 80 & 81 \\
\hline 12 & 2-OMe & 4-Me & & 120 & 84 \\
\hline 13 & 2-OMe & $3-\mathrm{NH}_{2}$ & & 120 & 74 \\
\hline
\end{tabular}

${ }^{\mathrm{a}}$ Reaction conditions: aryl iodides $(0.5 \mathrm{mmol})$, terminal alkynes $(0.75 \mathrm{mmol}), \mathrm{K}_{3} \mathrm{PO}_{4} \cdot 7 \mathrm{H}_{2} \mathrm{O}(1$ $\mathrm{mmol}), \mathrm{Pd}_{2}(\mathrm{dba})_{3}(0.5 \mathrm{~mol} \%), 80{ }^{\circ} \mathrm{C}$, under air. The reactions were monitored by TLC. ${ }^{\mathrm{b}}$ Isolated yields 
Table 3. Sonogashira reaction of aryl iodides with aliphatic terminal alkynes ${ }^{\mathrm{a}}$
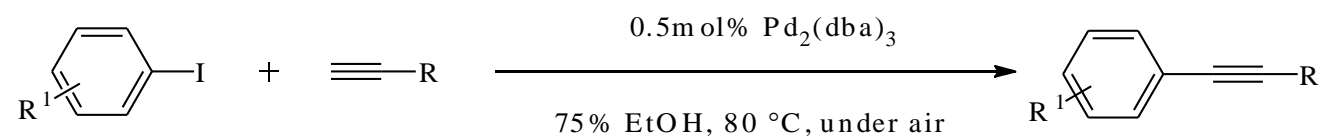

Entry 140

${ }^{\mathrm{a}}$ Reaction conditions: aryl iodides $(0.5 \mathrm{mmol})$, terminal alkynes $(0.75 \mathrm{mmol}), \mathrm{K}_{3} \mathrm{PO}_{4} \cdot 7 \mathrm{H}_{2} \mathrm{O}(1$ $\mathrm{mmol}), \mathrm{Pd}_{2}(\mathrm{dba})_{3}(0.5 \mathrm{~mol} \%), 80{ }^{\circ} \mathrm{C}$, under air. The reactions were monitored by TLC. ${ }^{\mathrm{b}}$ Isolated yields. ${ }^{\mathrm{c}} \mathrm{NaOH}$ was used in place of $\mathrm{K}_{3} \mathrm{PO}_{4} \cdot 7 \mathrm{H}_{2} \mathrm{O}$

\section{Conclusions}

In conclusion, we have developed a simple, highly efficient and phosphine-free environmentally benign protocol for the Sonogashira coupling of aryl iodides with aromatic or aliphatic terminal alkynes using a catalyst loading of $0.5 \mathrm{~mol} \%$ under aqueous, copper-free and aerobic conditions. Further investigation on the reaction mechanism and synthetic application of this protocol are underway in our laboratory 


\section{Experimental Section}

General. Unless otherwise noted, all the reactions were carried out under air. All aryl iodides and terminal alkynes were purchased from Alfa Aesar, Avocado and used without purification. Melting points were measured on a NOVEL R-3 microscopic digital melting point meter and were not corrected. ${ }^{1} \mathrm{H}$ NMR spectra were recorded on a Varian Inova 400 spectrometer. Chemical shifts are reported in ppm relative to TMS. ${ }^{13} \mathrm{C}$ NMR spectra were recorded at 100 MHz using TMS as internal standard. Mass spectroscopy data of the product were collected on a Micromass TOF mass spectrometer (EI, $70 \mathrm{eV}$ ). All products were isolated by short chromatography on a silica gel (200-300 mesh) column using mixtures of petroleum ether (60-90 ${ }^{\circ} \mathrm{C}$ ) and ethyl acetate as eluents. Compounds known were characterized by comparing their melting point, ${ }^{1} \mathrm{H}$ NMR spectra to the previously reported data. New compounds were characterized by melting point, ${ }^{1} \mathrm{H}$ NMR, ${ }^{13} \mathrm{C}$ NMR spectra and high resolution mass spectrometry.

\section{Typical experimental procedure for the Sonogashira reaction of aryl iodides with terminal} alkynes

A mixture of $\mathrm{Pd}_{2}(\mathrm{dba})_{3}(0.5 \mathrm{~mol} \%), \mathrm{K}_{3} \mathrm{PO}_{4} \cdot 7 \mathrm{H}_{2} \mathrm{O}(1 \mathrm{mmol})$, aryl iodides $(0.5 \mathrm{mmol})$, terminal alkynes $(0.75 \mathrm{mmol})$, ethanol $(3 \mathrm{~mL})$ and distilled water $(1 \mathrm{~mL})$ was stirred at $80{ }^{\circ} \mathrm{C}$ under air for the indicated time. After that, the mixture was added to brine $(15 \mathrm{~mL})$ and extracted three times with ethyl acetate $(3 \times 15 \mathrm{~mL})$. The solvent was concentrated under vacuum and the product was isolated by short chromatography on a silica gel (200-300 mesh) column.

3-((4-Methoxyphenyl)ethynyl)aniline (Table 2, entry 5). Brown solid. m.p.: 75-76 ${ }^{\circ} \mathrm{C} .{ }^{1} \mathrm{H}$ NMR: $\delta_{\mathrm{H}}(\mathrm{ppm}) 7.45\left(\mathrm{~d}, J=8.8 \mathrm{~Hz}, 2 \mathrm{H}, \mathrm{C}_{6} H_{4}\right), 7.12\left(\mathrm{t}, J=8.0 \mathrm{~Hz}, 1 \mathrm{H}, \mathrm{C}_{6} H_{4}\right), 6.92(\mathrm{~d}, J=7.6$ $\left.\mathrm{Hz}, 1 \mathrm{H}, \mathrm{C}_{6} H_{4}\right), 6.88-6.84\left(\mathrm{~m}, 3 \mathrm{H}, \mathrm{C}_{6} H_{4}\right), 6.65-6.63\left(\mathrm{~m}, 1 \mathrm{H}, \mathrm{C}_{6} H_{4}\right), 3.82\left(\mathrm{~s}, 3 \mathrm{H}, \mathrm{OCH}_{3}\right), 3.68$ $\left(\mathrm{s}, 2 \mathrm{H}, \mathrm{N} H_{2}\right) .{ }^{13} \mathrm{C} \mathrm{NMR}: \delta_{\mathrm{C}}(\mathrm{ppm}) 159.55\left(C_{6} \mathrm{H}_{4}\right), 146.26\left(C_{6} \mathrm{H}_{4}\right), 133.06\left(C_{6} \mathrm{H}_{4}\right), 129.27\left(C_{6} \mathrm{H}_{4}\right)$, $124.26\left(C_{6} \mathrm{H}_{4}\right), 121.96\left(C_{6} \mathrm{H}_{4}\right), 117.71\left(C_{6} \mathrm{H}_{4}\right), 115.48\left(C_{6} \mathrm{H}_{4}\right), 115.08\left(C_{6} \mathrm{H}_{4}\right), 113.98\left(C_{6} \mathrm{H}_{4}\right)$, $88.79(\mathrm{C} \equiv C), 88.29(C \equiv \mathrm{C}), 55.32\left(\mathrm{OCH}_{3}\right)$. MS (EI) $\mathrm{m} / z$ Calcd for $\mathrm{C}_{15} \mathrm{H}_{13} \mathrm{NO}$ : 223.0997. Found: $223.0990\left(\mathrm{M}^{+}\right)$.

1-(4-((3-Aminophenyl)ethynyl)phenyl)ethanone (Table 2, entry 10). Yellow solid. m.p.: 156$157^{\circ} \mathrm{C} .{ }^{1} \mathrm{H}$ NMR: $\delta_{\mathrm{H}}(\mathrm{ppm}) 7.93\left(\mathrm{~d}, J=8.0 \mathrm{~Hz}, 2 \mathrm{H}, \mathrm{C}_{6} H_{4}\right), 7.59\left(\mathrm{~d}, J=8.4 \mathrm{~Hz}, 2 \mathrm{H}, \mathrm{C}_{6} H_{4}\right), 7.15(\mathrm{t}$, $\left.J=8.0 \mathrm{~Hz}, 1 \mathrm{H}, \mathrm{C}_{6} H_{4}\right), 6.96\left(\mathrm{~d}, J=8.0 \mathrm{~Hz}, 1 \mathrm{H}, \mathrm{C}_{6} H_{4}\right), 6.88-6.87\left(\mathrm{~m}, 1 \mathrm{H}, \mathrm{C}_{6} H_{4}\right), 6.71-6.68(\mathrm{~m}$, $\left.1 \mathrm{H}, \mathrm{C}_{6} H_{4}\right), 3.72\left(\mathrm{~s}, 2 \mathrm{H}, \mathrm{NH}_{2}\right), 2.61\left(\mathrm{~s}, 3 \mathrm{H}, \mathrm{COCH}_{3}\right) .{ }^{13} \mathrm{C} \mathrm{NMR:} \delta_{\mathrm{C}}(\mathrm{ppm}) 197.36\left(\mathrm{COCH}_{3}\right)$, $146.38\left(C_{6} \mathrm{H}_{4}\right), 136.12\left(C_{6} \mathrm{H}_{4}\right), 131.70\left(C_{6} \mathrm{H}_{4}\right), 129.41\left(C_{6} \mathrm{H}_{4}\right), 128.34\left(C_{6} \mathrm{H}_{4}\right), 128.27\left(C_{6} \mathrm{H}_{4}\right)$, $123.33\left(C_{6} \mathrm{H}_{4}\right), 122.18\left(C_{6} \mathrm{H}_{4}\right), 117.83\left(C_{6} \mathrm{H}_{4}\right), 115.86\left(C_{6} \mathrm{H}_{4}\right), 93.05(\mathrm{C} \equiv C), 88.02(C \equiv \mathrm{C}), 26.63$ $\left(\mathrm{COCH}_{3}\right)$. MS (EI) $\mathrm{m} / z$ Calcd for $\mathrm{C}_{16} \mathrm{H}_{13} \mathrm{NO}$ : 235.0997. Found: $235.1000\left(\mathrm{M}^{+}\right)$.

3-((2-Methoxyphenyl)ethynyl)aniline (Table 2, entry 13). Brown sticky liquid. ${ }^{1} \mathrm{H} \mathrm{NMR}$ : $\delta_{\mathrm{H}}$ (ppm) $7.48\left(\mathrm{dd}, J=7.6,1.6 \mathrm{~Hz}, 1 \mathrm{H}, \mathrm{C}_{6} H_{4}\right), 7.31-7.27\left(\mathrm{~m}, 1 \mathrm{H}, \mathrm{C}_{6} H_{4}\right), 7.12(\mathrm{t}, J=8.0 \mathrm{~Hz}, 1 \mathrm{H}$, $\left.\mathrm{C}_{6} H_{4}\right), 6.98-6.88\left(\mathrm{~m}, 4 \mathrm{H}, \mathrm{C}_{6} H_{4}\right), 6.64\left(\mathrm{dd}, J=8.0,1.6 \mathrm{~Hz}, 1 \mathrm{H}, \mathrm{C}_{6} H_{4}\right), 3.90\left(\mathrm{~s}, 3 \mathrm{H}, \mathrm{OCH}_{3}\right), 3.65$ $\left(\mathrm{s}, 2 \mathrm{H}, \mathrm{N} H_{2}\right) .{ }^{13} \mathrm{C}$ NMR: $\delta_{\mathrm{C}}(\mathrm{ppm}) 159.95\left(C_{6} \mathrm{H}_{4}\right), 146.13\left(C_{6} \mathrm{H}_{4}\right), 133.59\left(C_{6} \mathrm{H}_{4}\right), 129.68\left(C_{6} \mathrm{H}_{4}\right)$, 
$129.19\left(C_{6} \mathrm{H}_{4}\right), 124.25\left(C_{6} \mathrm{H}_{4}\right), 122.24\left(C_{6} \mathrm{H}_{4}\right), 120.48\left(C_{6} \mathrm{H}_{4}\right), 118.00\left(C_{6} \mathrm{H}_{4}\right), 115.28\left(C_{6} \mathrm{H}_{4}\right)$, $112.58\left(C_{6} \mathrm{H}_{4}\right), 110.73\left(C_{6} \mathrm{H}_{4}\right), 93.70(\mathrm{C} \equiv C), 85.15(C \equiv \mathrm{C}), 55.86\left(\mathrm{OCH}_{3}\right)$. MS (EI) $\mathrm{m} / z$ Calcd for $\mathrm{C}_{15} \mathrm{H}_{13} \mathrm{NO}$ : 223.0997. Found: $223.1006\left(\mathrm{M}^{+}\right)$.

6-(4-Acetylphenyl)hex-5-ynenitrile (Table 3, entry 9). Light Yellow liquid. ${ }^{1} \mathrm{H}$ NMR: $\delta_{\mathrm{H}}(\mathrm{ppm})$ $7.78\left(\mathrm{~d}, J=8.4 \mathrm{~Hz}, 2 \mathrm{H}, \mathrm{C}_{6} H_{4}\right), 7.37\left(\mathrm{~d}, J=8.4 \mathrm{~Hz}, 2 \mathrm{H}, \mathrm{C}_{6} H_{4}\right), 2.53\left(\mathrm{t}, J=6.8 \mathrm{~Hz}, 2 \mathrm{H}, \mathrm{CH}_{2}\right)$, $2.48\left(\mathrm{~s}, 3 \mathrm{H}, \mathrm{COCH}_{3}\right), 2.47-2.45\left(\mathrm{~m}, 2 \mathrm{H}, \mathrm{CH}_{2}\right), 1.91-1.84\left(\mathrm{~m}, 2 \mathrm{H}, \mathrm{CH}_{2}\right) .{ }^{13} \mathrm{C} \mathrm{NMR}: \delta_{\mathrm{C}}(\mathrm{ppm})$ 197.22 $\left(\mathrm{COCH}_{3}\right), 135.98\left(C_{6} \mathrm{H}_{4}\right), 131.67\left(C_{6} \mathrm{H}_{4}\right), 128.17\left(C_{6} \mathrm{H}_{4}\right), 128.12\left(C_{6} \mathrm{H}_{4}\right), 119.17(C \equiv \mathrm{N})$, $90.87(\mathrm{C} \equiv C), 81.65(C \equiv \mathrm{C}), 26.57\left(\mathrm{COCH}_{3}\right), 24.38\left(\mathrm{CH}_{2}\right), 18.59\left(\mathrm{CH}_{2}\right), 16.20\left(\mathrm{CH}_{2}\right)$. MS (EI) $\mathrm{m} / z$ Calcd for $\mathrm{C}_{14} \mathrm{H}_{13} \mathrm{NO}$ : 211.0997. Found: $211.0997\left(\mathrm{M}^{+}\right)$.

1-(4-(5-Chloropent-1-yn-1-yl)phenyl)ethanone (Table 3, entry 10). Light Yellow liquid. ${ }^{1} \mathrm{H}$ NMR: $\delta_{\mathrm{H}}(\mathrm{ppm}) 7.88\left(\mathrm{~d}, J=8.4 \mathrm{~Hz}, 2 \mathrm{H}, \mathrm{C}_{6} H_{4}\right), 7.46\left(\mathrm{~d}, J=8.4 \mathrm{~Hz}, 2 \mathrm{H}, \mathrm{C}_{6} H_{4}\right), 3.71(\mathrm{t}, J=6.4$ $\left.\mathrm{Hz}, 2 \mathrm{H}, \mathrm{CH}_{2}\right), 2.64\left(\mathrm{t}, \mathrm{J}=6.8 \mathrm{~Hz}, 2 \mathrm{H}, \mathrm{CH}_{2}\right), 2.58\left(\mathrm{~s}, 3 \mathrm{H}, \mathrm{COCH}_{3}\right), 2.10-2.04\left(\mathrm{~m}, 2 \mathrm{H}, \mathrm{CH}_{2}\right) .{ }^{13} \mathrm{C}$ NMR: $\delta_{\mathrm{C}}(\mathrm{ppm}) 197.19\left(\mathrm{COCH}_{3}\right), 135.87\left(C_{6} \mathrm{H}_{4}\right), 131.67\left(C_{6} \mathrm{H}_{4}\right), 128.51\left(C_{6} \mathrm{H}_{4}\right), 128.16\left(C_{6} \mathrm{H}_{4}\right)$, $91.94(\mathrm{C} \equiv C), 80.95(C \equiv \mathrm{C}), 43.66\left(\mathrm{CH}_{2}\right), 31.23\left(\mathrm{CH}_{2}\right), 26.54\left(\mathrm{COCH}_{3}\right), 16.94\left(\mathrm{CH}_{2}\right) . \mathrm{MS}(\mathrm{EI})$ $\mathrm{m} / z$ Calcd for $\mathrm{C}_{13} \mathrm{H}_{13} \mathrm{ClO}: 220.0655$. Found: $220.0660\left(\mathrm{M}^{+}\right)$.

\section{Acknowledgements}

The authors thank the financial support from the National Natural Science Foundation of China (21076034, 20923006), the Fundamental Research Funds for the Central Universities (DUT11LK15), and the Ministry of Education (the Program for New Century Excellent Talents in University).

\section{References}

1. (a) Sonogashira, K.; Tohda, Y.; Hagihara, N. Tetrahedron Lett. 1975, 4467. (b) Sonogashira, K. J. Organomet. Chem. 2002, 653, 46. (c) Tykwinski, R. R. Angew. Chem. Int. Ed. 2003, 42, 1566. (d) Negishi, E.; Anastasia, L. Chem. Rev. 2003, 103, 1979. (e) Nicolaou, K. C.; Bulger, P. G.; Sarlah, D. Angew. Chem. Int. Ed. 2005, 44, 4442. (f) Chinchilla, R.; Nájera, C. Chem. Rev 2007, 107, 874. (g) Doucet, H.; Hierso, J.-C. Angew. Chem. Int. Ed. 2007, 46, 834.

2. (a) Dieck, H. A.; Heck, F. R. J. Organomet. Chem. 1975, 93, 259. (b) Cassar, L. J. Organomet. Chem. 1975, 93, 253.

3. Siemsen, P.; Livingston, R. C.; Diederich, F. Angew. Chem. Int. Ed. 2000, 39, 2633.

4. (a) Djakovitch, L.; Rollet, P. Adv. Synth. Catal. 2004, 346, 1782. (b) Cheng, J.; Sun, Y. H.; Wang, F.; Guo, M. J.; Xu, J. H.; Pan, Y.; Zhang, Z. G. J. Org. Chem. 2004, 69, 5428. (c) Li, J.-H.; Zhang, X.-D.; Xie, Y.-X. Eur. J. Org. Chem. 2005, 4256. (d) Gholap, A. R.; Venkatesan, K.; Pasricha, R.; Daniel, T.; Lahoti, R. J.; Srinivasan, K. V. J. Org. Chem. 2005, 
70, 4869. (e) Li, J.-H.; Liang, Y.; Xie, Y.-X. J. Org. Chem. 2005, 70, 4393. (f) Yi, C.; Hua, R. Catal. Commun. 2006, 7, 377. (g) Gu, Z.; Li, Z.; Liu, Z.; Wang, Y.; Liu, C.; Xiang, J. Catal. Commun. 2008, 9, 2154. (h) Komáromi, A.; Novák, Z. Chem. Commun. 2008, 4968. (i) Chandra, A.; Singh, B.; Khanna, R. S.; Singh, R. M. J. Org. Chem. 2009, 74, 5664.

5. Elangovan, A.; Wang, Y. H.; Ho, T. I. Org. Lett. 2003, 5, 1841.

6. (a) Alonso, D. A.; Nájera, C.; Pacheco, M. C. Org. Lett. 2000, 2, 1823. (b) Alonso, D. A.; Nájera, C.; Pacheco, M. C. Tetrahedron Lett. 2002, 43, 9365. (c) Kim, J. H.; Lee, D. H.; Jun, B. H.; Lee, Y. S. Tetrahedron Lett. 2007, 48, 7079. (d) Li, F.; Hor, T. S. A. Adv. Synth. Catal. 2008, 350, 2391. (e) Ray, L.; Barman, S.; Shaikh, M. M.; Ghosh, P. Chem. Eur. J. 2008, 14, 6646. (f) Lee, D. H.; Lee, Y. H.; Harrowfield, J. M.; Lee, I. M.; Lee, H. I.; Lim, W. T.; Kim, Y.; Jin, M. J. Tetrahedron 2009, 65, 1630. (g) Samantaray, M. K.; Shaikh, M. M.; Ghosh, P. J. Organomet. Chem. 2009, 694, 3477. (h) Bakherad, M.; Keivanloo, A.; Bahramian, B.; Hashemi, M. Tetrahedron Lett. 2009, 50, 1557.

7. (a) Herrmann, W. A. Angew. Chem. Int. Ed. 2002, 41, 1290. (b) Ma, Y. D.; Song, C.; Jiang, W.; Wu, Q. S.; Wang, Y.; Liu, X. Y.; Andrus, M. B. Org. Lett. 2003, 5, 3317. (c) Kantchev, E. A. B.; O'Brien, C. J.; Organ, M. G. Angew. Chem. Int. Ed. 2007, 46, 2768. (d) Marion, N.; Nolan, S. P. Acc. Chem. Res. 2008, 41, 1440.

8. (a) Urgaonkar, S.; Verkade, J. G. J. Org. Chem. 2004, 69, 5752. (b) Liang, B.; Dai, M.; Chen, J.; Yang, Z. J. Org. Chem. 2005, 70, 391. (c) Sajiki, H.; Zhang, G.; Kitamura, Y.; Maegawa, T.; Hirota, K. Synlett 2005, 1046. (d) Zhang, Y.; Shi, S. Synlett 2007, 1843. (e) Kawanami, H.; Matsushima, K.; Sato, M.; Ikushima, Y. Angew. Chem. Int. Ed. 2007, 46, 5129. (f) Mori, S.; Yanase, T.; Aoyagi, S.; Monguchi, Y.; Maegawa, T.; Sajiki, H. Chem. Eur. J. 2008, 14, 6994. (g) Thorwirth, R.; Stolle, A.; Ondruschka, B. Green Chem. 2010, 12, 985.

9. (a) Anderson, K. W.; Buchwald, S. L. Angew. Chem. Int. Ed. 2005, 44, 6173. (b) Li, J.-H.; Hu, X.-C.; Liang, Y.; Xie, Y.-X. Tetrahedron 2006, 62, 31. (c) Lipshutz, B. H.; Chung, D. W.; Rich, B. Org. Lett. 2008, 10, 3793. (d) Lee, D.-H.; Jung, J.-Y.; Jin, M.-J. Green Chem. 2010, 12, 2024. (e) Jin, M. J.; Lee, D. H. Angew. Chem. Int. Ed. 2010, 49, 1119. (f) Islam, M.; Mondal, P.; Roy, A.; Tuhina, K. Synthesis 2010, 2399. (g) Suzuka, T.; Okada, Y.; Ooshiro, K.; Uozumi, Y. Tetrahedron 2010, 66, 1064.

10. Liu, C.; Ni, Q. J.; Hu, P. P.; Qiu, J. S. Org. Biomol. Chem. 2011, 9, 1054. 